\title{
Dynamics of Magnetic Nanoparticles Studied by Neutron Scattering
}

Hansen, Mikkel Fougt; Bødker, Franz; Mørup, Steen; Lefmann, Kim; Clausen, Kurt Nørgaard; Lindgård, Per-Anker

Published in:

Physical Review Letters

Link to article, DOI:

10.1103/PhysRevLett.79.4910

Publication date:

1997

Document Version

Publisher's PDF, also known as Version of record

Link back to DTU Orbit

Citation (APA):

Hansen, M. F., Bødker, F., Mørup, S., Lefmann, K., Clausen, K. N., \& Lindgård, P-A. (1997). Dynamics of Magnetic Nanoparticles Studied by Neutron Scattering. Physical Review Letters, 79(24), 4910-4913.

https://doi.org/10.1103/PhysRevLett.79.4910

\section{General rights}

Copyright and moral rights for the publications made accessible in the public portal are retained by the authors and/or other copyright owners and it is a condition of accessing publications that users recognise and abide by the legal requirements associated with these rights.

- Users may download and print one copy of any publication from the public portal for the purpose of private study or research.

- You may not further distribute the material or use it for any profit-making activity or commercial gain

- You may freely distribute the URL identifying the publication in the public portal

If you believe that this document breaches copyright please contact us providing details, and we will remove access to the work immediately and investigate your claim 


\title{
Dynamics of Magnetic Nanoparticles Studied by Neutron Scattering
}

\author{
Mikkel F. Hansen, * Franz Bødker, and Steen Mørup \\ Department of Physics, Building 307, Technical University of Denmark, DK-2800 Lyngby, Denmark \\ Kim Lefmann, ${ }^{\dagger}$ Kurt N. Clausen, and Per-Anker Lindgård \\ Department of Condensed Matter Physics and Chemistry, Risø National Laboratory, DK-4000 Roskilde, Denmark
}

(Received 1 July 1997)

\begin{abstract}
We present the first triple-axis neutron scattering measurements of magnetic fluctuations in nanoparticles using an antiferromagnetic reflection. Both the superparamagnetic relaxation and precession modes in $\sim 15 \mathrm{~nm}$ hematite particles are observed. The results have been consistently analyzed on the basis of a simple model with uniaxial anisotropy and the Néel-Brown theory for the relaxation. [S0031-9007(97)04763-7]

PACS numbers: 75.50.Tt, 61.12.Ex, 76.60.Es, 78.70.Nx
\end{abstract}

The fluctuations of the magnetization direction in nanoparticles are currently subject to much attention. Studies of these phenomena are motivated not only by interest in the fundamental properties of ultrafine particles but also by the applications of such particles in, for example, magnetic recording media, ferrofluids, catalysts, etc. [1,2]. Most experimental studies of magnetic fluctuations in nanoparticles are carried out by use of ac and dc susceptibility measurements and by Mössbauer spectroscopy. Combining these techniques, it is possible to cover relaxation times in the range from more than $10^{4}$ to $10^{-9} \mathrm{~s}$. The superparamagnetic relaxation times have been found to vary with temperature in accordance with the Néel-Brown theory [3,4], apart from some observations at very low temperatures which suggest that macroscopic quantum tunneling may be the most important mechanism under these conditions [5]. At temperatures where the thermal energy is comparable to or larger than the magnetic anisotropy energy, the superparamagnetic relaxation time may be in the picosecond range. Apart from the superparamagnetic relaxation (fluctuations of the magnetization vector among the easy directions of magnetization), the magnetization vector also performs fast oscillations in the vicinity of the easy direction of magnetization (collective magnetic excitations) [6,7], with frequencies up to the $\mathrm{THz}$ range. The time scales of magnetization measurements and Mössbauer spectroscopy are too long to be used to measure these frequencies. In order to understand the full dynamics of the magnetization in nanoparticles, new experimental techniques are therefore needed.

Neutron scattering is sensitive to magnetic relaxation processes with characteristic times of the order of $10^{-10}$ $10^{-13} \mathrm{~s}$, but only few neutron studies of relaxation in nanoparticles have been carried out. Hennion et al. [8] studied magnetic relaxation in a sample of iron nanoparticles by small angle neutron scattering (SANS) and inelastic neutron scattering at small angles. They observed an influence of fluctuations of the magnetization near the easy direction of magnetization. In this paper, we have used triple-axis neutron scattering to study simultaneously superparamagnetic relaxation and collective magnetic excitations in nanoparticles of hematite $\left(\alpha-\mathrm{Fe}_{2} \mathrm{O}_{3}\right)$. Instead of using neutron scattering at small angles, we have studied the energy distribution of neutrons scattered at an angle corresponding to a purely magnetic diffraction peak. All of the data are fitted consistently using a simple model based on the assumption of uniaxial magnetic anisotropy.

Bulk hematite has the corundum structure and is antiferromagnetic below the Morin temperature $\left(T_{\mathrm{M}} \approx\right.$ $260 \mathrm{~K}$ ). Above this temperature, it is weakly ferromagnetic due to a small canting angle of the two sublattice moments. Magnetic resonance experiments on bulk material have revealed the presence of two excited magnetic states, which can be described on the basis of the hexagonal symmetry and the canting of the two magnetic sublattices [9]. In nanocrystalline hematite, $T_{\mathrm{M}}$ is less than $5 \mathrm{~K}$, and there is an effective, generally axial, magnetic anisotropy which is not present in the bulk. This anisotropy, which is large compared to the bulk anisotropy, is composed of a number of contributions e.g., surface anisotropy, stress anisotropy, etc. In the analysis of our results we have therefore not used an expression for the anisotropy which reflects the crystal symmetry. Instead, we have assumed a uniaxial anisotropy of the form

$$
E_{\text {an }}=-K_{\text {eff }} V \cos ^{2} \theta,
$$

where $K_{\text {eff }}$ is the effective magnetic anisotropy constant, $V$ is the particle volume, and $\theta$ is the angle between the sublattice magnetization and the easy direction.

The sample of nanocrystalline hematite was prepared by heating $250 \mathrm{~g} \mathrm{Fe}\left(\mathrm{NO}_{3}\right)_{3} \cdot 9 \mathrm{H}_{2} \mathrm{O}$ in an oven at temperatures slowly increasing from 60 to $90{ }^{\circ} \mathrm{C}$ over a period of 20 days. Mössbauer spectroscopy, electron microscopy, and x-ray diffraction confirmed that the sample consisted of nanocrystallites of hematite with a characteristic size of about $15 \mathrm{~nm}$ and a small amount of impurities $(\sim 10 \%)$ in the form of a structurally disordered component, probably ferrihydrite [10]. The magnetic moments of the particles may have contributions from both the 
canting of the two sublattices and uncompensated spins. However, Mössbauer studies showed that the sublattice magnetizations are oriented nearly perpendicular to applied fields larger than a few tesla [10]. This indicates that the former contribution is dominant.

The neutron scattering measurements were performed at DR3, Risø. We used the new cold source RITA spectrometer [11]. The spectrometer was configured in the conventional triple-axis mode with tight collimations, giving an energy resolution of $70 \mu \mathrm{eV}$ at an incident energy of $3.8 \mathrm{meV}$. The sample temperature was controlled in a displex cryostat or a furnace for the zero field experiments, and by a He-flow cryostat inside an Oxford Instruments $9 \mathrm{~T}$ magnet for experiments in applied fields. All data presented were taken at $q=1.37 \AA^{-1}$, corresponding to the antiferromagnetic (111) peak (rhombohedral indexing) from hematite, which was the strongest antiferromagnetic signal in our sample. In that way the influence from the disordered impurities was reduced to a small temperature-dependent increase of the background. In order to compare with a nonmagnetic signal, we took data at $q=1.1$ and at $1.9 \AA^{-1}$ as well, and observed no more than an elastic, incoherent background. It was established, from energy scans at other $q$ values close to the (111) peak and at the (100) antiferromagnetic peak, that the shape of the inelastic part of the energy spectra was independent of $q$, i.e., energy dispersion is absent, as expected for nanoparticles [12].

Figure 1 shows the logarithm of the normalized measured intensity versus energy transfer $\varepsilon$ for energy scans

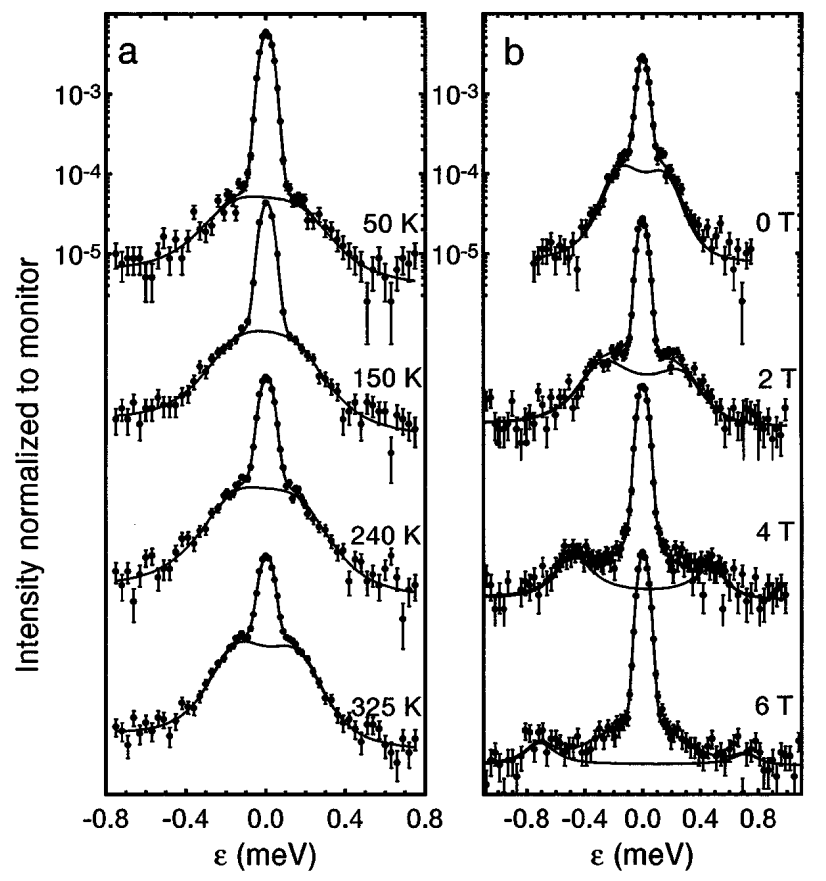

FIG. 1. (a) Typical inelastic neutron data at zero applied field. The solid lines represent fits as described in the text. (b) The same for $T=268 \mathrm{~K}$ in various applied fields. The asymmetry of the lines is due to a known geometrical effect. performed in zero applied field at the indicated temperatures [Fig. 1(a)] and at $268 \mathrm{~K}$ in applied fields [Fig. 1(b)]. From Fig. 1(a) it is clearly seen that the energy spectra are composed of a narrow component and a broad component and that the relative intensity of the latter increases with increasing temperature. A closer inspection of the broad component reveals that it has the shape of a smeared double peak. The splitting of this is seen to increase with increasing field [Fig. 1(b)] while the intensity decreases. These observations can be understood in terms of a simple picture. At low temperatures the sublattice magnetizations will perform collective magnetic excitations which can be described as precession states of the sublattice magnetizations in the effective anisotropy field combined with transitions between these precession states introduced by thermal agitation.

The lowest energy state is a uniform mode in which all spins in the two sublattices precess in phase. Transitions between the precession states, influencing the scattered neutrons, can therefore be understood in terms of excitation and deexcitation of spin waves with $q_{\mathrm{Sw}}=0$. The positions of the peaks at $\pm \varepsilon_{0}$ of the broad component are given by the energy differences to the neighboring precession states and thus depend on the effective anisotropy field.

Another dynamical process of the sublattice spins is the superparamagnetic relaxation. The typical time scale, $\tau$, of this process is given by $\tau=\tau_{0} \exp (\alpha)$ [3,4], where $\alpha \equiv K_{\text {eff }} V / k_{B} T$ and $\tau_{0} \sim 10^{-9}-10^{-12}$ s. This results in a quasielastic peak with a Lorentzian line shape. The fluctuations of the uniform in-phase mode yield maximum intensity for scattering near the (111) peak, and are perpendicular to the easy direction, while the fluctuations in the superparamagnetic relaxation are parallel to the easy direction.

Assuming a damped harmonic oscillator model for the dynamics of the precession states [13] the energy line shape, before convolution with the measured experimental resolution function, is

$$
\begin{aligned}
F(\varepsilon)= & A_{0} \delta(\varepsilon)+A_{1} \frac{D(\varepsilon)}{\pi} \frac{\Gamma}{\varepsilon^{2}+\Gamma^{2}} \\
& +A_{2} \frac{D(\varepsilon)}{\pi} \frac{2 \gamma \varepsilon_{0}^{2}}{\left(\varepsilon^{2}-\varepsilon_{0}^{2}\right)^{2}+4 \gamma^{2} \varepsilon^{2}}+C,
\end{aligned}
$$

where $A_{0}, A_{1}$, and $A_{2}$ are the areas of the incoherent elastic background, the quasielastic, and the inelastic components, respectively, $D(\varepsilon)=\varepsilon[n(\varepsilon)+1]$ is the detailed balance factor, $\Gamma$ is the HWHM of the Lorentz line, $\pm \varepsilon_{0}$ are the positions of the inelastic peaks, $\gamma$ is the HWHM of the inelastic peaks, and $C$ is the background. Neglecting the particle size distribution and interparticle interactions, the linewidth of the quasielastic peak is related to $\tau$ by $\Gamma=$ $\hbar / 2 \tau$ and $\varepsilon_{0}$ is equal to the energy difference $\Delta E$ between the precession states. The value of $A_{0}$ and its variation with temperature was estimated from energy scans at the nonmagnetic background performed at different 
temperatures. The results of the fits to Eq. (2) are seen as the full curves in Fig. 1.

Figures 2(a) and 2(b) show the relative area $A_{1} /\left(A_{1}+\right.$ $A_{2}$ ) and the linewidth $\Gamma$ of the quasielastic component as a function of temperature. Neglecting excitations to higher energy states [14], the areas $A_{1}$ and $A_{2}$ are expected to behave as $\left\langle S_{z}^{2}\right\rangle$ and $\left\langle S_{\perp}^{2}\right\rangle$, where the effective spin of the particles is composed of the "antisum" of the individual spins in the sublattices $a$ and $b: \vec{S}=\sum_{i}\left(\vec{s}_{i a}-\vec{s}_{i b}\right)$. Thus

$$
\frac{A_{1}}{A_{1}+A_{2}}=\left\langle\cos ^{2} \theta\right\rangle \text { and } \frac{A_{2}}{A_{1}+A_{2}}=\left\langle\sin ^{2} \theta\right\rangle,
$$

where $\langle\cdots\rangle$ is the thermal average. For the anisotropy energy of the form given by Eq. (1) we find $\left\langle\cos ^{2} \theta\right\rangle=$ $(2 \alpha)^{-1}[\exp (\alpha) / I(\alpha)-1]$, where $I(\alpha)=\int_{0}^{1} \exp (\alpha \times$ $\left.x^{2}\right) d x$. Based on this, a good fit to the data was obtained, as shown by the solid line in Fig. 2(a), with $K_{\text {eff }}^{T} V / k_{B}=700 \pm 200 \mathrm{~K}$. It should be noted that values deduced from the temperature variation, indicated by index $T$ in the following, also effectively include possible population of higher energy excitations [14]. To account for this, $K_{\text {eff }}^{T}$ is therefore expected to be smaller than the direct $K_{\text {eff }}$.

The width of the quasielastic component $\Gamma$ shown in Fig. 2(b) was fitted well with the Néel-Brown model yielding $K_{\text {eff }}^{\mathrm{NB}} V / k_{B}=500 \pm 200 \mathrm{~K}$ and $\tau_{0} \sim$ $7 \cdot 10^{-12} \mathrm{~s}$. The uncertainty of $\tau_{0}$ is rather large, probably about an order of magnitude. The value of $K_{\text {eff }}^{T} V / k_{B}$, obtained from the temperature dependence of the area ratios, is related to the fluctuations of the magnetization relatively near the energy minimum, whereas the value obtained from the superparamagnetic relaxation is related
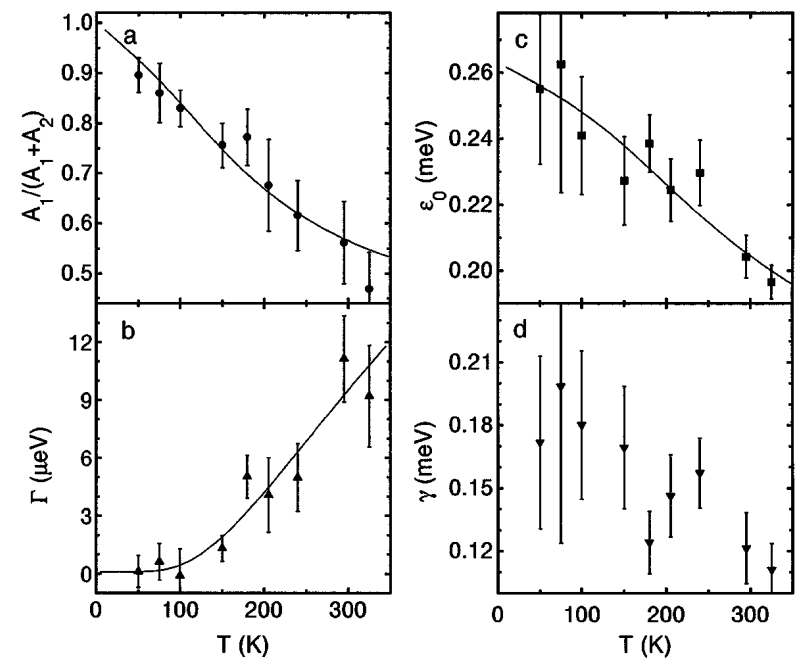

FIG. 2. (a) Relative intensities of the quasielastic peak. The solid line shows the calculated temperature dependence of $\left\langle\cos ^{2} \theta\right\rangle$. (b) The superparamagnetic relaxation parameter $\Gamma$, the line represents the Arrhenius law. (c) The position $\varepsilon_{0}$. (d) the linewidth $\gamma$ as a function of temperature. The lines indicate the fits described in the text. mainly to the height of the energy barrier. Yet, the two values agree quite well.

Figure 2(c) shows the values of $\varepsilon_{0}$ in zero field. It is slightly decreasing with increasing temperature, which suggests that the weighted average of the distance between the precession states decreases. To calculate the level splittings we write the quantum mechanical analog of Eq. (1) as $\mathcal{H}=-\kappa \sum_{i}\left(s_{i a z}^{2}+s_{i b z}^{2}\right)$, where $\kappa$ is the average axial anisotropy per site. Solving for an antiferromagnet with this anisotropy term we find for the uniform mode $[9,13]\langle\Delta E\rangle=\sqrt{\kappa s^{\prime}\left(\kappa s^{\prime}+g \mu_{B} B_{\mathrm{E}}\right)}\left\langle 2 S_{z}\right\rangle / S$, where $s^{\prime}=$ $s-\frac{1}{2}, s=\frac{5}{2}$ for $\mathrm{Fe}^{3+}$ and $B_{E}$ is the exchange field. The temperature dependence is approximately given by the average of the $z$ component of the large effective spin, which can be calculated using Eq. (1) as $\langle 2 \cos \theta\rangle=\alpha^{-1}[\exp (\alpha)-1] / I(\alpha)$. By fitting the temperature variation of $\varepsilon_{0}$, we obtained the value $K_{\text {eff }}^{T} V / k_{B}=1000 \pm 400 \mathrm{~K}$. From an analysis of the temperature dependence of the magnetic hyperfine splitting in the Mössbauer spectra due to collective excitations [6,7], the value $K_{\text {eff }}^{T} V / k_{B}=1000 \pm 200 \mathrm{~K}$ was found [10].

A direct measurement of the level splitting is obtained by the extrapolation of $\varepsilon_{0}$ to $T=0$ [Fig. 2(c)]. According to the above simple analysis, we find, using the bulk value $B_{\mathrm{E}}=927 \mathrm{~T}$ [9], the single site average anisotropy constant $\kappa / k_{B}=9.1 \pm 0.5 \times 10^{-4} \mathrm{~K}$. To compare with the total anisotropy energy, given by Eq. (1), for a particle with $N$ spins, we write $\mathcal{H}$ as $-N \kappa s^{2} \cos ^{2} \theta=-K_{\text {eff }} V \cos ^{2} \theta$. Assuming $N \approx$ $10^{5} \mathrm{Fe}^{3+}$ atoms for a $15 \mathrm{~nm}$ hematite nanoparticle, we obtain $K_{\text {eff }} V / k_{B} \approx 600 \mathrm{~K}$. This is very close to $K_{\text {eff }}^{T} V / k_{B}$ obtained from the thermal dependencies of the total effective spin $\vec{S}$. A precise average value for the particle size is needed to obtain the value for $K_{\text {eff }}$ calculated from the microscopically determined anisotropy $\kappa$. It should be noted that the inelastic energy $\varepsilon_{0}$ for the antiferromagnet is relatively high because the anisotropy is enhanced by the exchange field $\left(\varepsilon_{0} \propto \sqrt{\kappa B_{E}}\right)$. This causes the dynamical regimes of the inelastic and quasielastic phenomena to be well separated, and makes antiferromagnets particularly suited for neutron scattering. It is related to the linear energy dispersion for spin waves in antiferromagnets. The effect is not present in ferromagnets.

In summary, the temperature dependence of the relative areas of the quasielastic and inelastic components, the temperature dependence of $\varepsilon_{0}$, the width of the quasielastic component, and hyperfine field all yield values of the parameter $K_{\text {eff }}^{T} V / k_{B}$ which, within the uncertainty, are in agreement. The microscopically determined $K_{\text {eff }} V / k_{B}$, obtained from neutron scattering, is found to be in agreement as well.

The width of the inelastic component $\gamma$ [Fig. 2(d)] decreases slightly with increasing temperature. There are at least two contributions to the value of $\gamma$, namely, a distribution of $\Delta E$ and the finite lifetime of the states. 


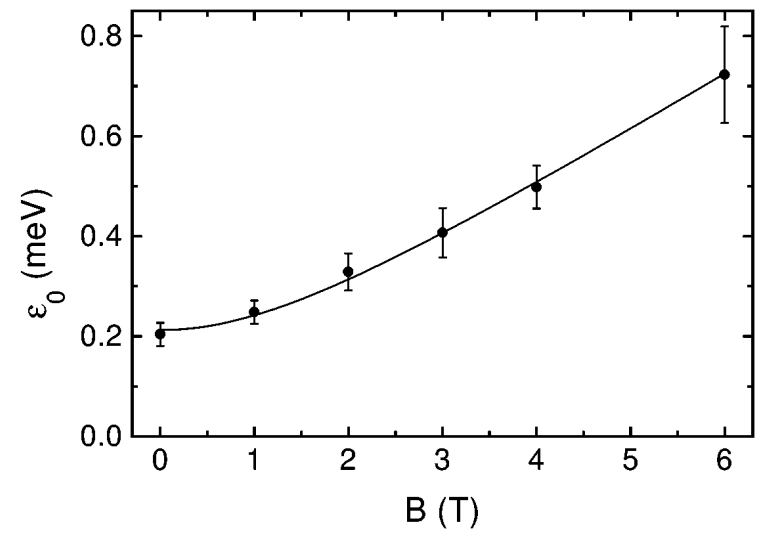

FIG. 3. Field variation of the outer peak position $\varepsilon_{0}(B)$ in Fig. 1(b). The line shows the fit described in the text.

If we had assumed for simplicity that the contribution to $\gamma$ due to the distribution of $\Delta E$ is negligible, and thus attributed the value of $\gamma$ to the lifetime, we would find a lower limit $\tau_{c}=4 \cdot 10^{-12} \mathrm{~s}$ for the lifetime of the precession states. The value of $\tau_{c}$ is probably related to, but smaller than, the value of $\tau_{0}$ because $1 / \tau_{c}$ is the typical frequency for transitions between the precession states, whereas $1 / \tau_{0}$ is the attempt frequency to jump over the energy barrier. According to Brown's model [4] for the relaxation time, $\tau_{0}$ increases with increasing temperature. If $\tau_{c}$ is proportional to $\tau_{0}$, the linewidth $\gamma=\hbar / 2 \tau_{c}$ should therefore decrease with increasing temperature.

For the spectra measured in applied fields a distribution of $\varepsilon_{0}$ reflecting the random orientation of the sublattices relative to the field is expected. As can be seen in Fig. 1(b), part of the inelastic component appears to be close to the zero field positions while two other clearly distinguishable peaks at $\pm \varepsilon_{0}(B)$ are shifted with the field $B$. For simplicity, we therefore assume a bimodal distribution representing particles with fully aligned moments along the field and particles only weakly influenced by the field. For the latter, we held $\varepsilon_{0}$ and $\gamma$ close to the values obtained in zero field. The resulting fits are shown as the lines in Fig. 1(b).

Figure 3 shows the variation of $\varepsilon_{0}(B)$ as a function of the applied magnetic field. It is seen that $\varepsilon_{0}(B)$ increases when $B$ increases. $\varepsilon_{0}(B)$ has contributions from both the anisotropy energy and the applied field. If the particles contributing to the outer peak are assumed to have their ferromagnetic moments aligned with the external field, the anisotropy field, $B_{\text {an }}=\varepsilon_{0} / g \mu_{B}$, will be perpendicular to the applied field and the resulting effective field is $B_{\text {eff }} \approx \sqrt{B_{\text {an }}^{2}+B^{2}}$ giving $\varepsilon_{0}(B)=g \mu_{B} B_{\text {eff }}$. By fitting the data in Fig. 3 with this model, we find that $g \mu_{B}=$ $0.12 \pm 0.01 \mathrm{meV} / \mathrm{T}$, in agreement with the theoretical value $g \mu_{B}=0.116 \mathrm{meV} / \mathrm{T}$ as expected for $\mathrm{Fe}^{3+}(g=$ 2). Apart from shifting the inelastic peaks to higher energies, the applied field also results in a decrease in their relative area and a decreasing linewidth of the quasielastic peak. This is explained by the fact that the field reduces the amplitude of the fluctuations near the energy minimum and also slows down the superparamagnetic relaxation.

It is the first time that the fluctuations of the magnetization in magnetic nanoparticles have been studied using an antiferromagnetic reflection. Utilizing this reflection we have been able to study the superparamagnetic relaxation and the collective magnetic excitations simultaneously, and make a clear distinction between the two types of dynamics. The interpretation of the results was confirmed by the temperature and field variation of the spectra.

We thank Danish Council for Natural Sciences and the Danish Council for Technical Research for their support.

*Electronic address: mfhansen@fysik.dtu.dk

†Electronic address: kim.lefmann@ risoe.dk

[1] See, for example, Magnetic properties of Fine Particles, edited by J. L. Dormann and D. Fiorani (North-Holland, Amsterdam, 1992); Nanophase Materials: Synthesis Properties - Applications, edited by G.C. Hadjipanayis and R. W. Siegel (Kluwer, Dordrecht, 1994).

[2] J. L. Dormann, D. Fiorani, and E. Tronc, Adv. Chem. Phys. 98, 183 (1997).

[3] L. Néel, Ann. Geophys. 5, 99 (1949).

[4] W. F. Brown, Jr., Phys. Rev. 130, 1677 (1963).

[5] E. M. Chudnovsky, J. Appl. Phys. 73, 6697 (1993); B. Barbara et al., ibid 73, 6703 (1993); L. Thomas et al., Nature (London) 383, 145 (1996).

[6] S. Mørup and H. Topsøe, Appl. Phys. 11, 63 (1976).

[7] S. Mørup, J. Magn. Magn. Mater. 37, 39 (1983).

[8] M. Hennion et al., Europhys. Lett. 25, 43 (1994).

[9] A.H. Morrish, Canted Antiferromagnetism: Hematite (World Scientific, Singapore, 1994).

[10] F. Bødker et al. (to be published).

[11] T.E. Mason, K. N. Clausen, G. Aeppli, D. F. McMorrow, and J. K. Kjems, Can. J. Phys. 73, 697 (1995).

[12] P. V. Hendriksen, S. Linderoth, and P.-A. Lindgård, Phys. Rev. B 48, 7259 (1993).

[13] See, for example, P.-A. Lindgård, in Spin Waves and Magnetic Excitations 1, edited by A. S. Borovik-Romanov and S. K. Sinha (Elsevier, New York, 1988), p. 287.

[14] From the measured spin wave spectrum for bulk hematite [E. J. Samuelsen and G. Shirane, Phys. Status Solidi 42, 241 (1970)], the lowest energy $q \neq 0$ excitations in the nanoparticles are estimated (as in Ref. [12]) to be at and above $\sim 10 \mathrm{meV}$ and therefore weakly populated in the temperature range considered. Population of the antiphase antiferromagnetic $q=0$ mode [9] (not observable in the present scans) is also expected. The experiments showed that $A_{1}+A_{2}$ dropped by $14 \%$ from $T=50 \mathrm{~K}$ to $T=325 \mathrm{~K}$, slightly more than the thermal decrease of the squared sublattice magnetization in bulk $\alpha-\mathrm{Fe}_{2} \mathrm{O}_{3}$. 